\title{
Computational modeling of the atomizing of a gas stream from a pneumatic nozzle
}

\author{
Kuznetsov V.A., Shebelev A.V., Minakov A.V. \\ Siberian Federal University \\ Krasnoyarsk, Russian Federation \\ victor_partner@mail.ru
}

\begin{abstract}
Atomizing of liquid is widely used in modern technology. Atomizing occupies an extremely important place in power engineering, when burning liquid fuels. In this work provides verification of the method for modeling the atomizing of a gasliquid stream from a pneumatic nozzle. Primary atomization is modelled using Large Eddy Simulation model (LES) and The Volume Of Fluid model (VOF). The results of the calculations show an acceptable agreement with experiment on the main characteristics of the spray. The results of calculations can be very useful for estimations, since they allow qualitatively correct determination of the structure of a two-phase flow, parameters and shape of droplets, and the speed and direction of their motion, which in principle can not be obtained by other methods.
\end{abstract}

Keywords-pneumatic nozzle; primary break-up; volume of fluid (VOF) model; large eddy simulation (LES) model; CFD

\section{INTRODUCTION}

Atomizing of liquid is widely used in modern technology [1-2]. Atomizing occupies an extremely important place in power engineering, when burning liquid fuels. Ways of burning are very diverse. However, for all methods, the fuel atomization stage is mandatory, since by repeatedly increasing the total contact surface of the media, strengthening the heat exchange of the crushed fuel with the gaseous medium and improving the mixing of the fuel particles with the oxidant, significantly contributes to the intensification of the combustion process.

The enormous importance of atomizing the liquid for solving a wide range of technical problems led to the creation of a large number of various nozzle designs meeting the specific requirements of the tasks being solved. The organization of gas flows plays a decisive role in the efficiency of the pneumatic nozzle. Thereby the task is to develop an effective and reliable numerical method for describing the atomizing of liquid fuels in perspective burners.

A review of existing methods for simulating the spraying of liquids has shown that in these models the droplets generation is simplified resulting in inaccurate and unrealistic simulations. $[3,4]$. For example, the blob atomization method which is the most employed model not only simply generates parcels with the size of the nozzle diameter but also does not take into account the physics of in-nozzle turbulence [5-7]. This method does not represent a detailed physical and satisfying modeling of the relevant processes during primary break-up. These constraints motivate the study of the primary break-up modeling method using the VOF/LES approach.

Volume of Fluid (VOF) methods are useful for free surface flows and have originally be developed by Hirt et al. [8]. These methods are efficient to handle complex interfaces as internal moving boundaries. In work [8-11] show a simple, but powerful, method is described that is based on the concept of a fractional volume of fluid (VOF). This method is shown to be more flexible and efficient than other methods for treating complicated free boundary configurations.

Large eddy simulation (LES) directly resolves large scales and models small scales [2, 12]. Modelling only small scales and solving the large scales, allows the use of a much coarser mesh and larger time steps in LES compared with Direct Numerical Simulation (DNS). Despite this, LES still needs a finer mesh compared with the ones used for Reynolds Averaged Navier Stokes (RANS) computations.

The aim of this work is the verification of the method for modeling the atomizing of a gas-liquid stream from a pneumatic nozzle on based on experimental data.

\section{PROBLEM STATEMENT AND RESEARCH METHODS}

For verification the numerical methodology for modeling the primary break-up, the following problem was considered [4]. The geometry and dimensions of the nozzle are shown in Fig.1. Dimensions of the nozzle are as follows: $d_{l}=2.9 \mathrm{~mm}, D_{l}$ $=3.8 \mathrm{~mm}, D_{g}=5.6 \mathrm{~mm}$. Width of annular clearance $0,7 \mathrm{~mm}$. Water is supplied through the central channel. Air is fed through the annular channel. In experiments, the water velocity varied in the range from $0,15-1,5 \mathrm{~m} / \mathrm{s}$. Air speed $-15-250 \mathrm{~m} / \mathrm{s}$.

In the calculations, four spray variants were considered corresponding to the parameters presented in Table 1. Weber's number was determined by the standard method (1):

$$
W e=\rho_{l}\left(U_{l}-U_{g}\right)^{2} D_{l} / \sigma
$$




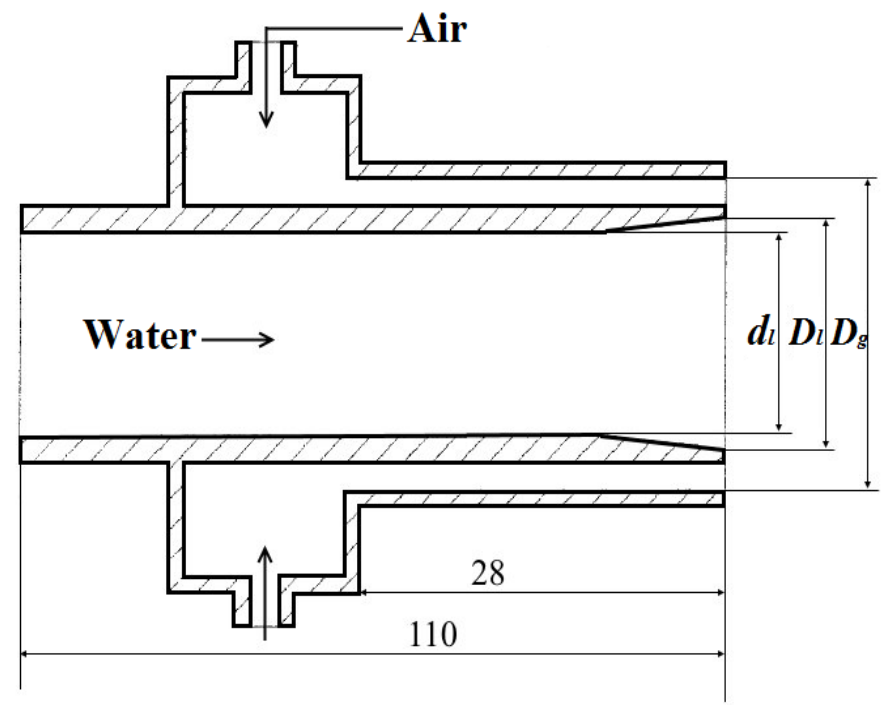

Fig.1. The geometry of the nozzle (mm).

The geometry of the computational space is shown in Fig.2. For calculations, a Cartesian grid was used with detailing at the nozzle walls. The computational mesh is shown in Fig. 3. The computational mesh was 820000 cells.

\section{TABLE I. CALCULATION OPTIONS}

\begin{tabular}{|c|c|c|}
\hline № & Air velocity, Ug, $\mathbf{M} / \mathbf{c}$ & Weber number, We \\
\hline 1 & 28 & 52 \\
\hline 2 & 40 & 110 \\
\hline 3 & 80 & 200 \\
\hline 4 & 120 & 800 \\
\hline
\end{tabular}

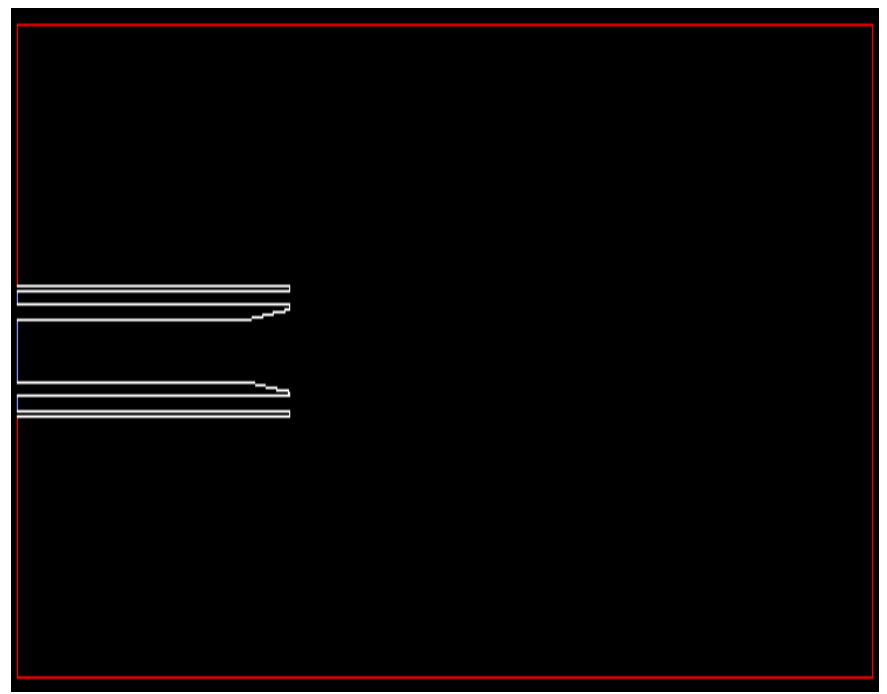

Fig.2. The computational space.

Primary atomization is modelled using the volume of fluid model. The VOF model can model two or more immiscible fluids by solving a single set of momentum equations and tracking the volume fraction of each of the fluids throughout the domain. To simulate turbulence, we used Large Eddy Simulation model. Large eddy simulation (LES) directly resolves large scales and models small scales. Modelling only small scales and solving the large scales, allows the use of a much coarser mesh and larger time steps in LES compared with Direct Numerical Simulation (DNS). Large eddy simulation (LES) therefore falls between DNS and RANS in terms of the fraction of the resolved scales. For closure, the WALE model was used. The time step was determined based on the condition $C F L=2$. Such a restriction leads to the fact that the magnitude of the time step is $3 \times 10^{-7} \mathrm{~s}$. The mean time for calculating the flow of order $0,3 \mathrm{~s}$.

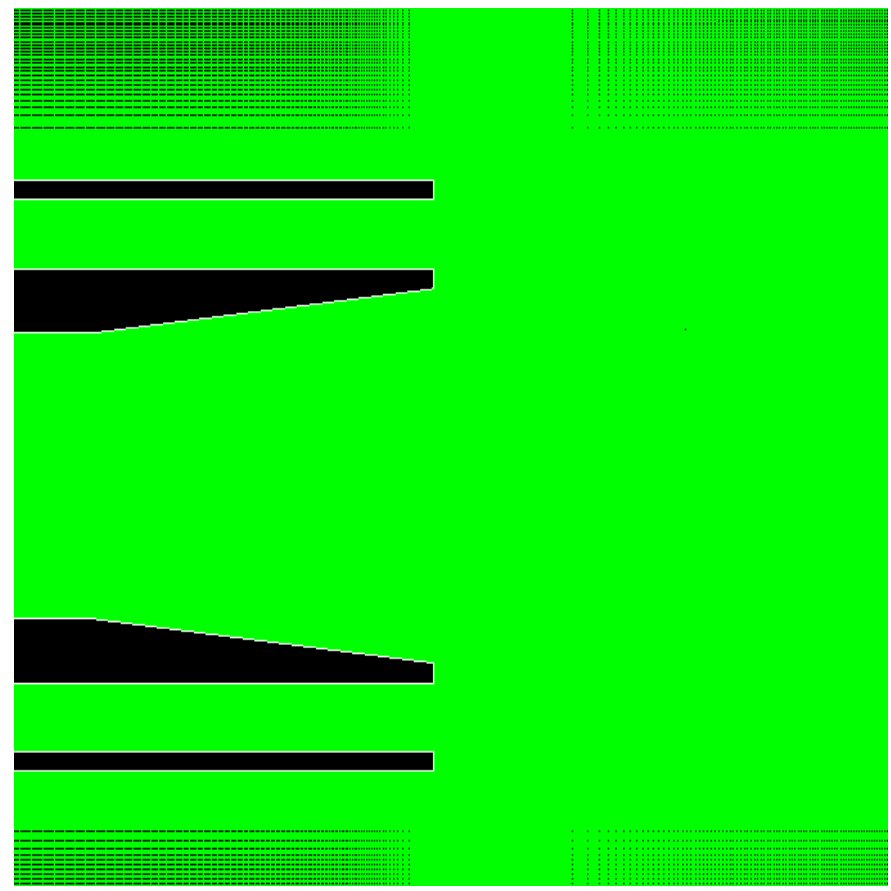

Fig.3. The computational mesh near the nozzle.

\section{RESULTS AND DISCUSSION}

In Figures 4-7 for the considered variants, a qualitative comparison of the calculated atomizing results with experimental photographs is given [13]. The figures show the instantaneous distribution of the volume fraction of the liquid in the calculated cells. From the comparison, a good qualitative agreement between calculation and experiment is observed. The calculation correctly describes the shape and length of the liquid jet, the angle of the atomizing, the shape and the approximate size of the resulting ligaments. A visual analysis of the atomizing quality showed that the average size of the droplets formed in the calculations approximates the observed droplet diameter in the experiments.

For the variant $\mathrm{Ug}=120 \mathrm{M} / \mathrm{c}, \mathrm{We}=800$ in Fig. 8 shows the distribution of the time-averaged volume fraction of liquid. 


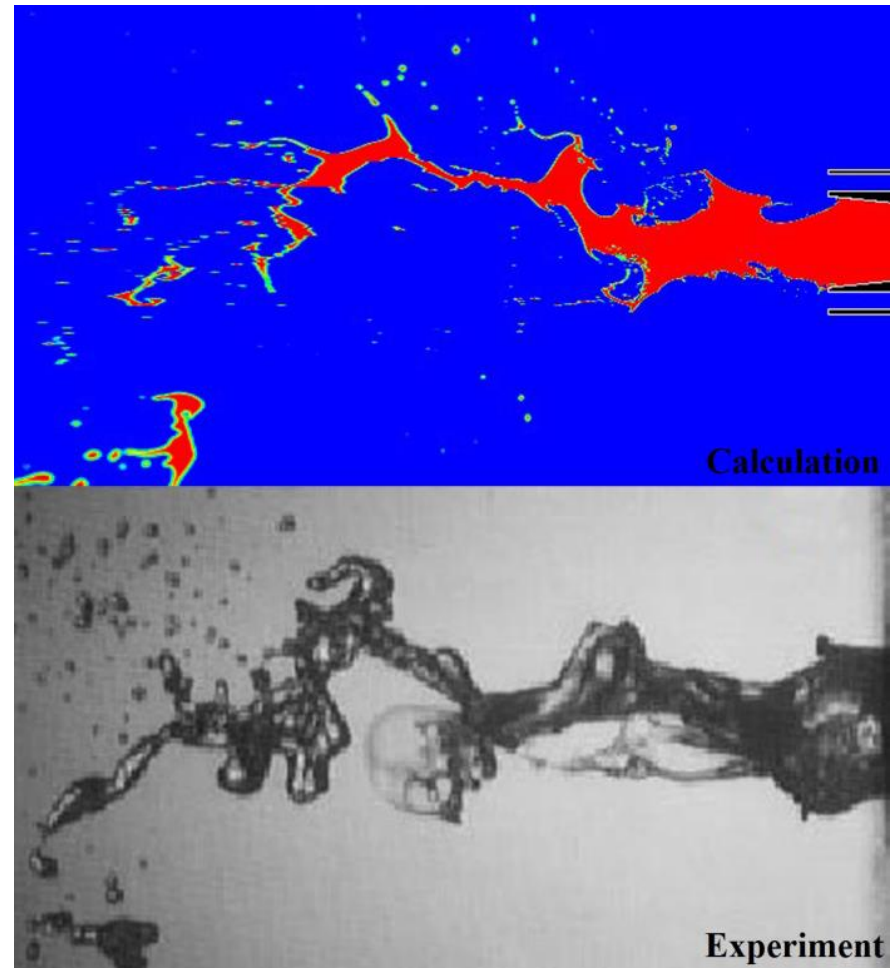

Fig.4. Instantaneous flow visualization of the jet break-up $(U g=28 \mathrm{M} / \mathrm{c}$, $\mathrm{We}=52$ ).
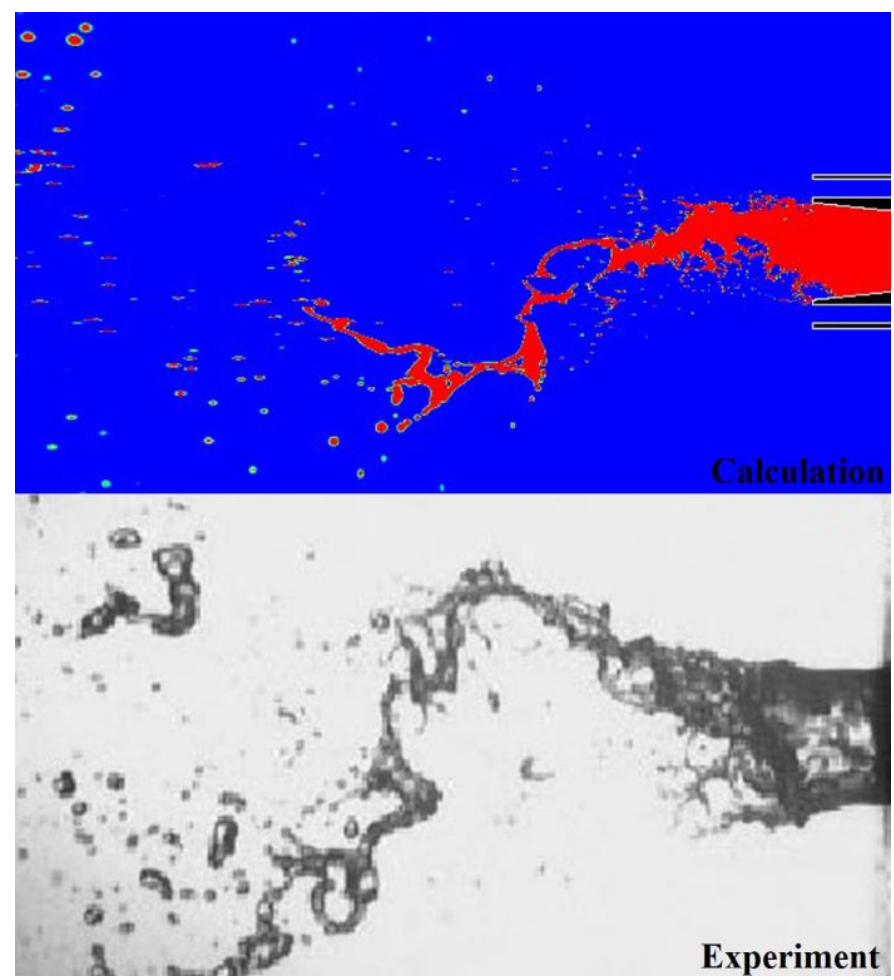

Fig.5. Instantaneous flow visualization of the jet break-up $(\mathrm{Ug}=40 \mathrm{M} / \mathrm{c}$, $\mathrm{We}=110$ ).

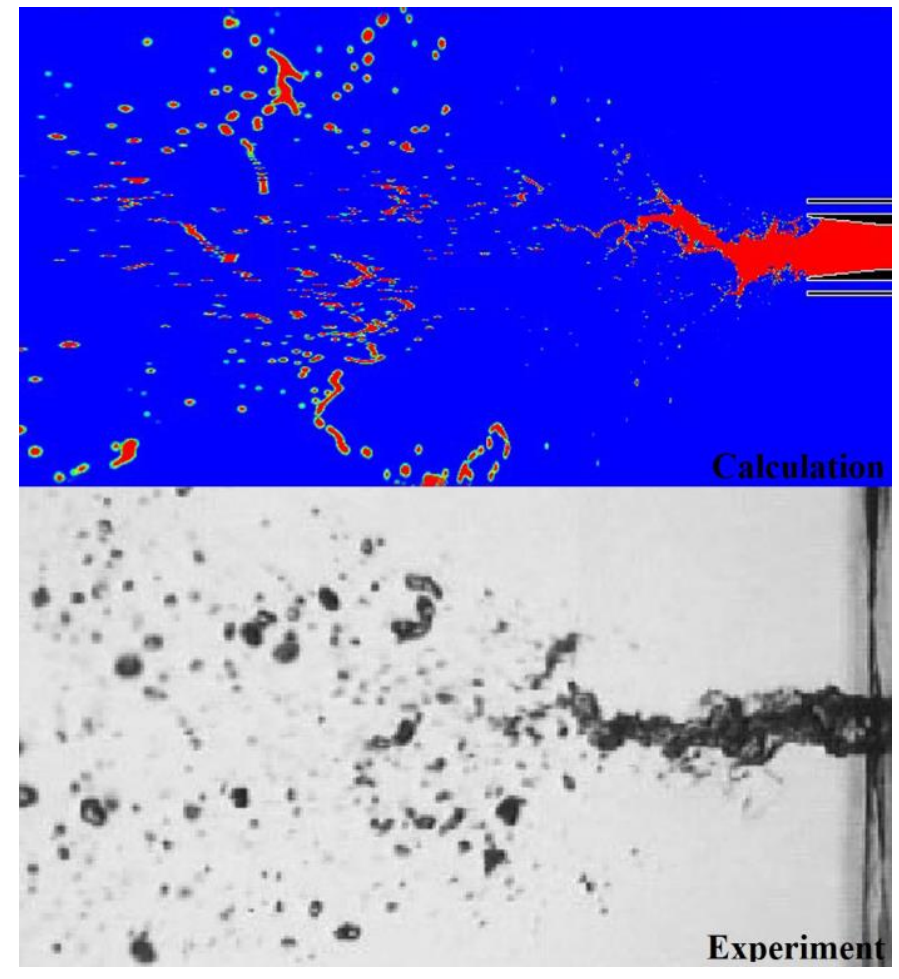

Fig.6. Instantaneous flow visualization of the jet break-up $(\mathrm{Ug}=80 \mathrm{~m} / \mathrm{c}$, $\mathrm{We}=200$ ).

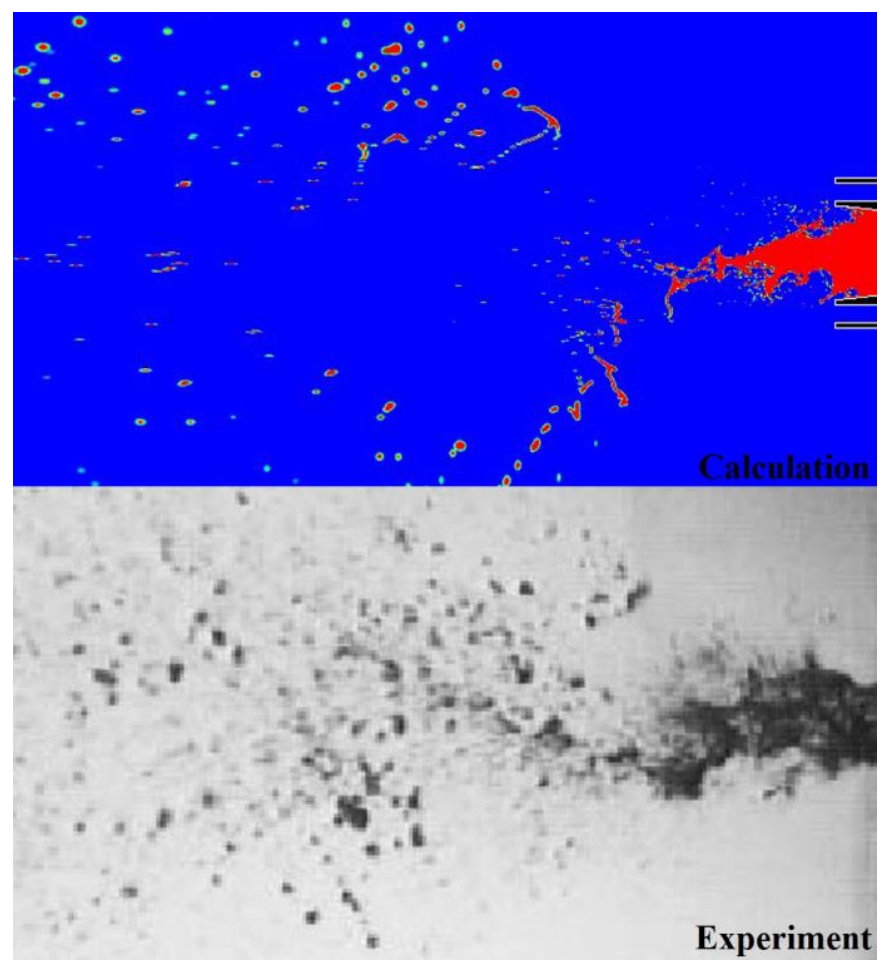

Fig.7. Instantaneous flow visualization of the jet break-up $(\mathrm{Ug}=120 \mathrm{M} / \mathrm{c}$, $\mathrm{We}=800$ ) 


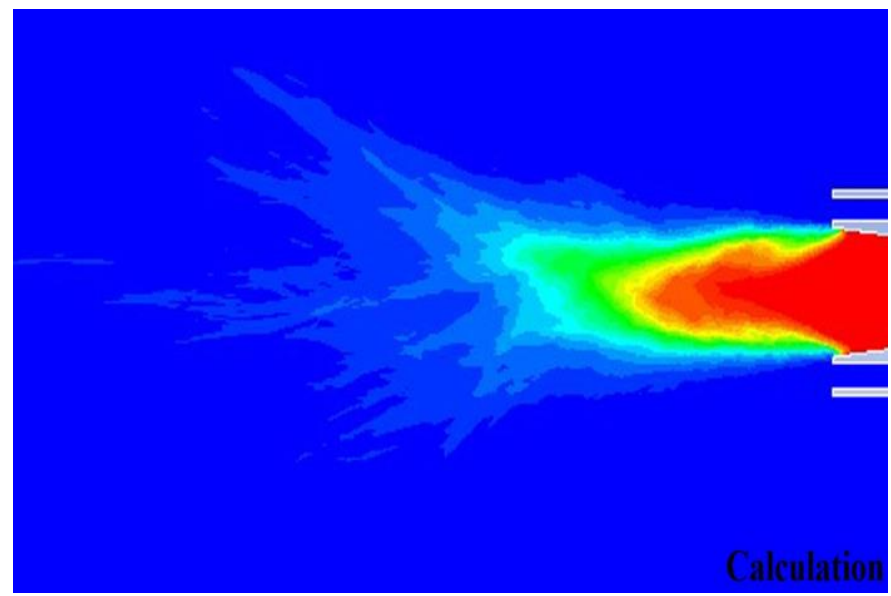

Fig.8. Averaged flow visualization of the jet break-up

\section{CONCLUSION}

We have performed verification of the method for modeling the spraying of a gas-liquid stream from a pneumatic nozzle. The results of the calculations show an acceptable agreement with experiment on the main characteristics of the spray. A visual analysis of the atomizing quality showed that the average size of the droplets formed in the calculations approximates the observed droplet diameter in the experiments. The results of calculations can be very useful for estimations, since they allow qualitatively correct determination of the structure of a two-phase flow, parameters and shape of droplets, and the speed and direction of their motion, which in principle can not be obtained by other methods.

\section{REFERENCES}

[1] Lightfoot, M. D. A., A Fundamental Classification of Atomization Processes, OMB No. 0704-0188, 2008.

[2] L. Bravo et. all, Numerical Investigation of Liquid Jet Breakup and Droplet Statistics with Comparison to X-ray Radiography, Propulsion and Energy Forum, July 25-27, 2016, Salt Lake City, UT

[3] Bong C.H., Numerical and experimental analysis of diesel spray dynamics including the effects of fuel viscosity, University of Tasmania, 2010 .

[4] Kaario, O., Vuorinen, V., Hulkkonen, T., Keskinen, K., Nuutinen, M., Larmi, M. \& Tanner, F. X., Large Eddy Simulation of High Gas Density Effects in Fuel Sprays, Atomization and Sprays, 23, No.4, 2013

[5] REITZ, R. D. (1987). "Modeling atomization processes in high-pressure vaporizing sprays".Atomization and Spray Technology, vol. 3, pp. 309337.

[6] Reitz R. D, Diwakar R (1987). "Structure of High-Pressure Fuel Sprays". SAE-paper 870598.

[7] Baumgarten, C. (1996). "Mixture Formation in Internal Combustion Engines". ISBN -103-540-30835 -0 Springer-Verlag Berlin Heidelberg New York.

[8] C. Hirt, B. Nichols. Volume of fluid (VOF) method for the dynamics of free boundaries, J Comput Phys, 39 (1981), pp. 201-225

[9] Francis H. Harlow and J. Eddie Welch, Numerical calculation of timedependent viscous incompressible flow of fluid with free surface, Phys Fluids, 8 (12) (1965), pp. 2182-2189

[10] A 3d unsplit forward/backward volume-of-fluid approach and coupling to the level set method, J Comput Phys, 233 (2013), pp. 10-33

[11] D. Fuster, et al. Simulation of primary atomization with an octree adaptive mesh refinement and VOF method, Int J Multiphase Flow, 35 (2009), pp. 550-565

[12] S.V. Apte a,*, M. Gorokhovski b,1, P. Moin, LES of atomizing spray with stochastic modeling of secondary breakup, International Journal of Multiphase Flow 29 (2003), pp. 1503-1522

[13] Lasheras J.C., Villermaux E., Hopfinger E.J., "Break-up and atomization of a round water jet by a high-speed annular air jet," - J. Fluid Mech. (1998), vol. 357, pp. 351-379.. 